\title{
Blastare? No, grazie
}

Vera Gheno

PUBBLICATO: 01 LUGLIO 2019

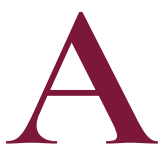

ll'incirca dal 2016, è andato diffondendosi in rete un impiego particolare di un verbo che precedentemente era già diffuso in contesti giovanili, seppure con un significato un po' diverso: blastare. È uno dei tanti verbi "ibridi" formati da un sostantivo inglese con aggiunta della desinenza della prima coniugazione -are (l'unica produttiva: cfr. le schede precedentemente pubblicate su questo sito su googlare, twittare e whatsappare). Il significato con cui si trova usato oggi il verbo è piuttosto preciso: indica l'uso di attaccare, deridere o zittire, con violenza e pubblicamente, solitamente da una posizione di forza e nel contesto dei social network, chi ha detto o scritto una evidente sciocchezza.

Blastare deriva dal verbo inglese to blast 'far esplodere, far brillare, distruggere, far saltare in aria'; il verbo, in inglese, è usato anche in senso figurato: il Merriam-Webster riporta il significato di attack vigorously, cioè 'attaccare con violenza', e come esempio d'uso la frase "blasting their opponents in the media" 'attaccando con violenza i loro oppositori sui mezzi di comunicazione di massa'; gli Oxford Dictionaries specificano che esiste un uso informale di to blast nel significato dicriticize fiercely 'criticare aspramente' e forniscono l'esempio "the school was blasted by government inspectors" la scuola venne criticata aspramente dagli ispettori del governo'.

Come accennato in apertura, il verbo blastare non è alla sua prima apparizione in italiano. Ne abbiamo varie attestazioni nel corso degli ultimi decenni, transitando da vari gerghi. La prima occorrenza a cui siamo riusciti a risalire è del novembre 1988 , sulla copertina di un periodico dedicato ai videogiochi, Zzap!: "Armalyte: blastiamoli vivi!".

Qui l'uso è nel senso proprio di 'annientare il nemico'. Già nel 1983 era stato diffuso sul mercato un videogioco arcade chiamato proprio Blaster, in cui lo scopo era per l'appunto far esplodere gli avversari (il demo, cioè la breve sequenza dimostrativa all'inizio del gioco, si chiudeva proprio con la frase Blast or be blasted!). Il nome, con varianti, ritorna poi in giochi degli anni successivi, fino a identificare un vero e proprio sottogenere di videogiochi (quelli in cui, fondamentalmente, l'avversario andava fatto esplodere). L'uso del verbo italianizzato sembra, insomma, diretta conseguenza della tendenza inglese di quegli anni.

Successivamente, nel 1993, ritroviamo il verbo usato ancora in senso proprio nel testo di un brano degli Articolo 3I, "Strade di città":

Dal fondo della strada bagliori di una deflagrazione nella notte, il nome di ciascuno appare ovunque, rumore di catene rotte [...] blastano la gabbia! Strada di città.

L'esempio è riportato anche in Renzo Ambrogio, Giovanni Casalegno, Scrostati gaggio! Dizionario storico dei linguaggi giovanili, Torino, Utet libreria, 2004, p. 4I, che cita anche la forma sblastare con il significato di 'distruggere, far saltare'.

Pochi anni dopo, il verbo compare ancora nel linguaggio giovanile, in particolare in quello dell'area romana, acquisendo un senso figurato: si rinviene un'attestazione del 2008 in Slangopedia con il 
significato di 'prendere in giro, deridere, sfottere con decisione, o render palese l'altrui torto con cinismo, senza alcuna pietà' e in Bruttastoria, altro repertorio di giovanilismi, nel 2013, chiosato come 'deridere, umiliare, demolire, usare sarcasmo verso una persona'. Entrambi questi esempi sono citati da Licia Corbolante sul suo blog Terminologia nella ricostruzione della storia del termine, che risale al febbraio 2017 .

Mentre i due usi summenzionati esistono anche in inglese, la successiva specializzazione del verbo a indicare la violenta esposizione al pubblico ludibrio della stupidità altrui, in particolare da una posizione di forza e sui social network, è specifica dell'italiano: seguendo all'incirca lo stesso procedimento già visto per influencer, è stato preso un verbo inglese dal significato più generico e lo si è sottoposto a una restrizione semantica nell'ambito del gergo della rete. Particolarmente rilevante, per la popolarizzazione del verbo, è risultato l'accostamento con un comportamento ricorrente da parte del giornalista Enrico Mentana (comportamento che ha fatto nascere anche gruppi Facebook dedicati al suo "culto", per esempio Enrico Mentana blasta laggente) risalente, come rileva Licia Corbolante sul suo blog, al 2016 circa, anno a cui risale questo esempio:

Blastare, per chi non lo sapesse, significa prendere in giro, deridere, umiliare senza pietà ed è un adattamento fai-da-te al verbo inglese blast e il direttore sta svolgendo il suo nuovo compito egregiamente. Populisti, troll, complottisti ed esperti della domenica sono tutti allertati: Mentana vi distruggerà (Enrico Stefanini, Enrico Mentana che umilia i suoi hater: una pagina Facebook bellissima, Dailybest.it, I/8/2016)

Nel corso degli anni sono assurti agli onori della cronaca anche altri personaggi pubblici che indulgono nella pratica con altrettanta visibilità, quindi l'esempio del giornalista non è certo l'unico, anche se conserva la sua rilevanza di... capostipite della tendenza.

Come spesso accade, il verbo ha attecchito abbastanza diffusamente perché indica un preciso comportamento che si nota soprattutto in rete, ed è difficilmente sostituibile se non con perifrasi: non è schernire, non è deridere, non è svergognare, non è umiliare; è una sorta di unione di tutti i comportamenti summenzionati, con in più l'aggravante che l'azione avviene specificamente da una posizione di forza: l'esperto che blasta l'inesperto, il colto che blasta l'ignorante, lo scienziato che blasta il credulone ecc.

Il verbo blastare compare 26.900 volte su Google, in contesti italiani; dal 2015 a oggi, c'e stata una costante progressione nel numero di occorrenze, da 915 a I.680 (2016) a 2.550 (2017) a 4.I70 (2018) per arrivare a $5.28 \mathrm{o}$ al $2 \mathrm{I}$ aprile 2019. Il verbo non compare nei dizionari, ma ricorre solo nella sezione Neologismi del Vocabolario Treccani, con datazione 20I8; è di fatto assente dai quotidiani. Lo troviamo solo su Repubblica, con 4 occorrenze di cui tre, datate 2011 e 20I5, con il "vecchio" significato, e solo una, del 20I9, con il nuovo. Ė invece molto usato - non a caso - sui social network, a ulteriore riprova della sua circolazione piuttosto specifica - seppure non esclusiva - a quel contesto. Un picco nell'uso del verbo è avvenuto il ıs aprile zorg e nei giorni immediatamente successivi. Quel giorno, la persona addetta alla cura del profilo Facebook "INPS per la famiglia" ha iniziato a rispondere in maniera decisamente sarcastica a utenti che stavano ingolfando la pagina dell'ente con domande spesso molto ingenue. Il fatto ha provocato un'ingente quantità di discussioni sui social network e su varie testate giornalistiche, portando al proliferare sia delle occorrenze del verbo sia alle domande in merito alla sua origine e al suo significato.

Il verbo ha attechito al punto che esiste anche il sostantivo blastatore, ossia chi blasta, e il participio presente blastante usato come aggettivo, in espressioni quali comportamento blastante. 
Il comportamento blastante ha, al momento, molti seguaci: chi blasta, infatti, viene spesso osannato come il campione della "tribù", come la "persona che non le manda a dire". Ciò nonostante, a osservare meglio, si può notare che blastare non porta a conseguenze positive sul lungo periodo: rafforzerà l'accordo di chi era già dello stesso parere del blastatore, ma non farà quasi sicuramente cambiare idea a chi è stato blastato, che, anzi, tenderà a rifugiarsi ancora di più tra coloro che la pensano come lui, formando una nicchia pressoché impenetrabile a idee divergenti: una cosiddetta "camera dell'eco" o echo chamber. Dunque, sarebbe meglio evitare di cedere alla tentazione di blastare e cercare altre strade per controbattere a chi esprime il proprio dissenso o la propria ignoranza in maniera rumorosa o scomposta.

\section{Cita come:}

Vera Gheno, Blastare? No, grazie, "Italiano digitale", 2019, IX, 2019/2 (aprile-giugno)

DOI: $10.35948 / 2532-9006 / 2020.3095$

\section{Copyright 2019 Accademia della Crusca}

Pubblicato con licenza creative commons CC BY-NC-ND 